\title{
Commentary
}

In this issue of the Journal of Neurosciences in Rural Practice, Chakrabarti et al..$^{[1]}$ report a rare case of undifferentiated high-grade pleomorphic sarcoma of breast presenting with metastatic spread to the brain. The case highlights important issues faced by pathologists in the recognition of metastatic lesions in addition to imparting a vital lesson for clinical neuroscience practitioners.

Metastatic tumors are the commonest brain malignancies and their incidence is possibly increasing as survival times improve for many extra-cranial neoplasms. ${ }^{[2]}$ Despite advances in radiological techniques, tissue diagnosis may sometimes be required even in patients with a known primary site. This may happen, for instance, if $\mathrm{CT}$ and non-contrast MRI do not conclusively exclude non-neoplastic mimics like abscesses, infections, demyelinating diseases and vascular lesions. ${ }^{[3]}$ Alternatively, imaging features may not be typical of brain metastases or could be discordant with the primary site diagnosis. Sometimes, one may wish to ascertain the nature of the brain lesion if spread occurs unexpectedly in a long-term survivor whose systemic cancer is otherwise well-controlled. ${ }^{[3,4]}$
In contrast to the above, in patients without a known primary site, it becomes vital to identify the brain lesion with certainty. This happened in the reported case $^{[1]}$ and is not an uncommon situation overall, as it has been estimated that up to $30 \%$ of brain metastases are either diagnosed synchronously with the primary tumor or are actually detected before the primary site (precocious presentation) ${ }^{[3-5]}$ In these patients, CT chest/abdomen, mammography and FDG-PET are useful to pin-point the primary site but further extensive evaluation is inappropriate in the absence of specific symptoms or any indications from the brain biopsy, whether open or stereotactic. ${ }^{[6]}$ Histopathology of the brain metastasis, though nearly always difficult to obtain, provides valuable clues in indicating a likely organ of origin. Immunohistochemical staining demonstrating antigen(s) specific for the tissue, organ or neoplasm of origin can be the clinching supplementary investigation. Cerebrospinal fluid cytology with cytospin examination can confirm the coexistence of carcinomatous or leukemic meningitis. Histological sub-typing is also important in planning therapy as it distinguishes relatively radio/chemo-sensitive tumors like small cell carcinoma lung, leukemia/lymphoma or germ cell tumors from ones unlikely to benefit from these modalities. ${ }^{[3-5]}$ 
Cytopathological examination, whether in the form of fine needle aspiration cytology of solid masses or exfoliative cytology of body cavities has evolved tremendously and is now a well established and distinct diagnostic discipline. Most Indian neuropathologists are expert regional cytopathologists too, accustomed as they are to interpreting squash and touch preparations of CNS tissues for rapid and cost-effective intra-operative diagnosis. ${ }^{[7]}$ Although, the role of cytology in CNS lesions is restricted, it is tempting to envisage clinical situations wherein even stereotactic biopsies may be unsafe (e.g. in thrombocytopenic patients, or those with coagulopathies) or with lesions in close proximity to vital structures where fine needle aspiration cytology, due to its minimally invasive nature could be definitively diagnostic. For instance, in cases where the extracranial primary site is documented histologically, cytology may be all that is needed for confirmation of the suspected metastasis.

Multidisciplinary teams require clinicians to work in tandem with pathologists and the best neurosurgeons have an in-depth understanding of both the strengths and weaknesses of anatomic pathologic diagnosis. In the case described by Chakrabarti and colleagues, ${ }^{[1]}$ the primary diagnosis in the breast mass was rendered additionally challenging as FNAC of soft tissue tumors is prone to well-documented diagnostic pitfalls. ${ }^{[8]}$ This is due to the general rarity of these tumors, their propensity for cellular pleomorphism and to undergo necrosis and myriad secondary changes like calcification, hyalinization, degeneration etc., as well as due to the importance of recognizing architectural patterns in subtyping of these lesions. ${ }^{[8]}$ This last information is unfortunately mostly unavailable on aspirate smears, although cell blocks and microbiopsies may compensate to some extent. In the setting of a breast mass, the present authors also had to exclude commoner mammary tumors with mesenchymal components like cystosarcoma phyllodes and sarcomatoid carcinoma in their differential diagnosis.

And finally, but perhaps most importantly, the report by Chakrabarti et al..$^{[1]}$ reminds us how a detailed medical history and complete physical examination can yield rich dividends in patients with neurological symptoms. In the present case, eliciting the history of the breast mass avoided expensive and time-consuming work-up with complicated interpretations and clinical-radiological correlations that would otherwise almost certainly have been ordered. Truly, the history and physical examination remain excellent "diagnostic tests" in the astute clinician's armamentarium even in this era of high-resolution imaging and molecular subclassifications of neoplasms.

\section{Prashant Sharma}

Department of Haematology, Postgraduate Institute of Medical Education and Research, Chandigarh, India

Address for correspondence: Dr. Prashant Sharma, Department of Haematology, Research Block A, Postgraduate Institute of Medical Education and Research, Chandigarh, India. E-mail: sharma.prashant@pgimer.edu.in

\section{References}

1. Chakrabarti I, Ghosh N, Giri A. Cytologic diagnosis of undifferentiated high grade pleomorphic sarcoma of breast presenting with brain metastasis. J Neurosci Rural Pract 2013;4:188-90.

2. Lu-Emerson C, Eichler AF. Brain metastases. Continuum (Minneapolis Minn) 2012;18:295-311.

3. Soffietti R, Cornu P, Delattre JY, Grant R, Graus F, Grisold W, et al. Brain Metastases. In: Gilhus NE, Barnes MP, Brainin M, editors. European Handbook of Neurological Management. Vol. 1, $2^{\text {nd }}$ ed. London: Blackwell Publishing; 2011. p. 437-45.

4. Tsao MN, Rades D, Wirth A, Lo SS, Danielson BL, Vichare A, et al. International practice survey on the management of brain metastases: Third international consensus workshop on palliative radiotherapy and symptom control. Clin Oncol (R Coll Radiol) 2012;24:e81-92.

5. Patel TR, Knisely JP, Chiang VL. Management of brain metastases: Surgery, radiation, or both? Hematol Oncol Clin North Am 2012;26:933-47.

6. Stošić-Opinćal T. Diagnostics of central nervous system metastatic disease. Arch Oncol 2006;14:41-3.

7. Goel D, Sundaram C, Paul TR, Uppin SG, Prayaga AK, Panigrahi MK, et al. Intraoperative cytology (squash smear) in neurosurgical practice-pitfalls in diagnosis experience based on 3057 samples from a single institution. Cytopathology 2007;18:300-8.

8. Layfield LJ. Cytopathology of bone and soft tissue tumors. Oxford: Oxford University Press; 2002.

\begin{tabular}{|l|l|}
\hline \multicolumn{2}{|c|}{ Access this article online } \\
\hline Quick Response Code: & \\
\hline & Website: \\
\hline
\end{tabular}

